\title{
Faire dessiner le terrain
}

La nature à « risques » et les jardins de subsistance de Tanna et Tongoa (Vanuatu)

To Make drawing the field. Risky nature and subsistence gardens of Tongoa and Tanna (Vanuatu)

\section{Maëlle Calandra}

\section{(2) OpenEdition}

Journals

Édition électronique

URL : https://journals.openedition.org/tc/6822

DOI : $10.4000 /$ tc. 6822

ISBN : $1952-420 X$

ISSN : 1952-420X

Éditeur

Éditions de l'EHESS

\section{Édition imprimée}

Date de publication : 19 juin 2013

Pagination : 182-201

ISBN : 978-2-7351-1637-9

ISSN : 0248-6016

Référence électronique

Maëlle Calandra, «Faire dessiner le terrain », Techniques \& Culture [En ligne], 60 | 2013, mis en ligne le

19 juin 2016, consulté le 29 septembre 2022. URL : http://journals.openedition.org/tc/6822 ; DOI :

https://doi.org/10.4000/tc.6822 


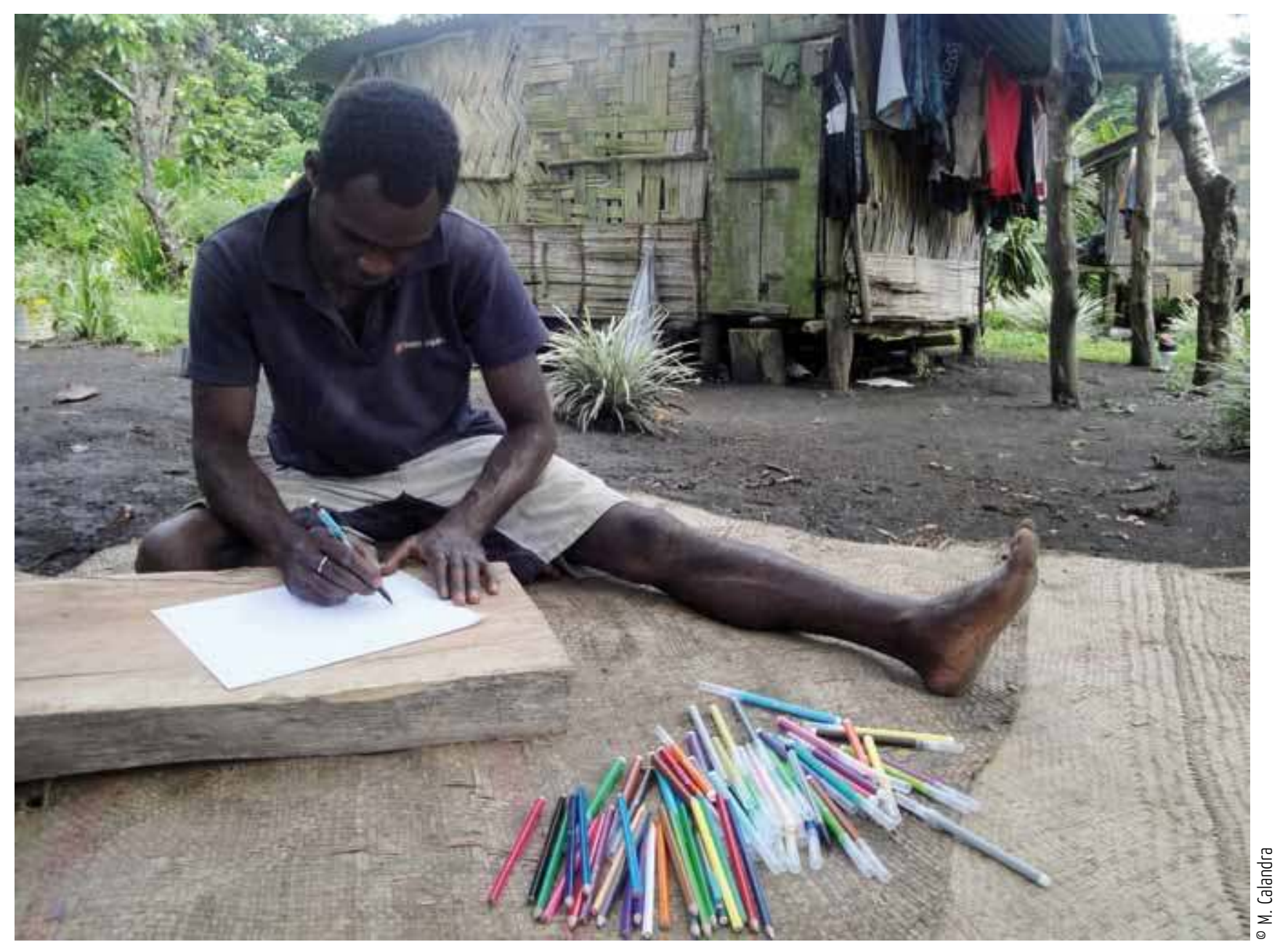




\section{FAIRE DESSINER LE TERRAIN}

\section{La nature à « risques » et les jardins de subsistance de Tanna et Tongoa (Vanuatu)}

Se fondant sur deux terrains ethnographiques relatifs à des questions de nature ${ }^{1}$, cet article cherche à montrer l'intérêt méthodologique du dessin, véritable outil de production de données à Tanna et Tongoa au Vanuatu. Ce processus de figuration est un médiateur pertinent dans la mesure où il met en lumière les relations qu'entretiennent les individus avec leurs espaces de vie et les représentations qu'ils s'en font (Pagézy, Carrière \& Sabinot, 2010 : 10). Les dessins du terrain sont également des vecteurs d'informations grâce aux commentaires qu'ils suscitent. Ils permettent à l'ethnographe, en contournant les difficultés du discours lorsque le sujet évoqué provoque le malaise des informateurs, d'accéder à des informations nouvelles et complémentaires. À partir d'un corpus ${ }^{2}$ d'entretiens et de dessins, il s'agit ici d'interroger le dessin en tant que médium entre l'individu et son environnement, mais aussi en tant qu'interface entre le terrain et le chercheur.

\section{Une ethnographie dessinée}

L'une des spécificités de la démarche anthropologique réside dans la relation directe du chercheur avec un terrain, sorte de « laboratoire de l'altérité [...] tant sont multiples les interlocuteurs et variées leurs positions dans le dispositif de recherche mis en place » (Vidal, 2010 : 19). La pratique du terrain implique nécessairement des relations, puisqu'il y est question d'individus et que s'y joue la rencontre entre l'ethnographe et 
ses interlocuteurs. L'ethnographie privilégie l'observation participante, car l'immersion prolongée du chercheur dans une culture et une société donnée permet, comme le signalait Bronislaw Malinowski, d'évaluer plus précisément « le point de vue de l'indigène, ses rapports avec la vie, de comprendre sa vision de son monde [...] c'està-dire que nous devons étudier la prise que la vie a sur lui » (2010 [1922] : 81-82). À Tongoa et Tanna (Vanuatu), en 2011 et 2012, dans le cadre de mes travaux ethnographiques sur les rapports à la nature et au paysage dans un milieu insulaire soumis aux « risques », j'ai choisi, outre l'observation participante, les entretiens et la photographie, de recourir à un outil de production de données alternatif: le dessin. Si elle a jusqu'à présent été peu utilisée, cette technique d'enquête n'est pas nouvelle. Au xix siècle lors de l'expédition menée dans le détroit de Torrès en Papouasie Nouvelle-Guinée, Alfred Cort Haddon (zoologue et anthropologue britannique) avait fait dessiner, aux enfants et aux adultes de l'île de Mer, des masques et des figures importantes de leur mythologie (Haddon, 2010 [1935]). À la fin des années 1930, l'ethnologue Thérèse Rivière proposait également aux Ath Abderrahman de l'Aurès (Algérie) de tracer sur des feuilles de dessin, des scènes de la vie ordinaire qu'elle compila et nomma par la suite « l'album de dessins indigènes » (Coquet, 2009 : 189). Plus récemment, Michèle Cros a recouru à cette technique pour faire représenter le SIDA et la maladie en pays Lobi burkinabé (1993). En Papouasie Nouvelle-Guinée, Martin Soukup (2011) a demandé aux Yawan de dessiner ce qu'évoquait pour eux le mot « culture».

Contrairement à la photographie, le dessin n'est pas l'enregistrement d'une situation lumineuse à un instant donné (Aumont, 2005 [1990] : 125), ni une image figée, mais le résultat d'un ensemble de techniques et d'actions graphiques permettant de rendre reconnaissable une image observée ou imaginée sur un support d'inscription (toile, feuille de papier, sable, mur, etc.). Cette action de figuration comme l'explique Philippe Descola, est avant tout un acte pensé grâce auquel:

" Un objet matériel quelconque est investi de façon ostensible d'une "agence" (au sens de l'anglais agency) socialement définie à la suite d’une action de façonnage, d'aménagement, d'ornementation ou de mise en situation visant à lui donner un potentiel d'évocation iconique d'un prototype réel ou imaginaire qu'il dénote de façon indicielle (par délégation d'intentionnalité) en jouant sur une ressemblance directe de type mimétique ou sur tout autre type de motivation identifiable de façon médiate ou immédiate » (2006: 167).

Je fais ici l'hypothèse que ces images dessinées, par ce qu'elles laissent transfigurer, constituent un nouveau médium pour interroger les cosmologies locales et dans ce contexte particulier, les rapports avec un environnement soumis aux désastres. Selon l'historien de l'art Pierre Francastel, le processus créatif à la base de la représentation graphique est rendu possible parce qu'il nomme la " pensée figurative » (1967). Pour reprendre les mots de Fabio La Rocca, la perception visuelle est « un engagement actif de l'esprit, un esprit qui met en acte le processus d'abstraction en transformant le perçu en notion et catégorie de la pensée » (2007 : 34). Aussi, les dessins, puisqu'ils résultent de la main de l'homme, peuvent être considérés comme un produit social et acquièrent un intérêt sociologique lorsqu'ils sont intégrés comme données dans le projet de recherche. 


\section{La nature en images}

L'archipel du Vanuatu est situé dans le Pacifique sud, sur la « ceinture de feu $»^{3}$ entre les plaques pacifique et indo-australienne, exposant ainsi le pays à une activité volcanique et sismique importante. Néanmoins, le risque le plus menaçant de novembre à avril est celui des cyclones tropicaux ${ }^{4}$. En raison des phénomènes naturels auxquels leur île est exposée, les habitants des îles de Tongoa, située au centre de l'archipel (village de Kurumape), et de Tanna, localisée au sud du pays (village de Lamlu) entretiennent des rapports particuliers avec leur environnement. L'élément central de leur écologie locale est un volcan. À Tongoa, le volcan Tompuku est sous-marin tandis qu'à Tanna, le volcan Yasur est aérien. Ces volcans apparaissent comme des figures symboliques paradoxales, à la fois source de vie et de chaos. Les populations considèrent en effet qu'après avoir décimé les générations passées lors d'éruptions volcaniques importantes, ces derniers assurent à présent, par le « feu », la pérennité de celles à venir, en ayant rendu la nature prolifique et les ressources halieutiques abondantes. Pour maintenir des relations réciproques avec ces volcans, véritables acteurs socialisés, et permettre une forme de cohabitation, une mémoire générationnelle et sensorielle nourrie de savoirs empiriques spécifiques continue d'être transmise à travers les histoires coutumières relatant leurs origines. Face au danger que représentent la proximité du volcan et l'existence d'autres phénomènes climatologiques et géophysiques également destructeurs (séismes, cyclones, érosion constante des côtes et éruptions volcaniques), les habitants semblent avoir élaboré un jeu d'équilibre entre la crainte et la confiance au travers d'un système de connaissances naturalistes empiriques complexes. Hérité des anciens, celui-ci est pensé comme un mécanisme de prédiction et par extension, de contrôle des risques. Par une lecture attentive des phénomènes naturels récurrents, les insulaires affirment pouvoir les prévoir, les expliquer et les doter de sens au regard de leur mythologie. Les phénomènes à risques auxquels Tongoa et Tanna sont exposées s'inscrivent dans une logique de répétition ayant permis aux habitants d'intégrer à leur système de représentations et d'actions, les scénarios futurs de la catastrophe. Cette hypothèse paraît confirmer les suggestions de Mondher Kilani concernant « la certitude de la catastrophe » (2007: 50).

En 2011 à Tongoa, c'est suite à plusieurs entretiens au sujet du volcan sous-marin, lors desquels l'histoire coutumière du volcan, sa forme et ses couleurs ainsi que sa présence au fond de l'eau étaient décrites et justifiées que l'idée de faire dessiner le volcan aux informateurs m'est apparue. Tous semblaient connaître sa forme et son étendue sous-marine, pourtant invisible depuis la surface, puisqu'il est situé à soixante mètres de profondeur. Afin de voir comment ils pouvaient le représenter je proposais, après chaque entretien, des feuilles blanches, des feutres et des crayons de couleur. Je demandais que soit dessiné le volcan sur une première feuille blanche et, sur une seconde, le souvenir d'une éruption passée. Une fois le matériel de dessin remis, je précisais que ce n'était pas l'esthétique du dessin qui m'intéressait, mais sa qualité descriptive. Les dessins achevés, j'inscrivais au dos des feuilles: l'âge, le sexe de l'illustrateur et le titre qu'il donnait au dessin, mais aussi la date, le lieu de réalisation et parfois des annotations sur les figurations (par exemple le nom des végétaux représentés). Ils constituaient un outil intéressant pour susciter des interactions et des commentaires spontanés. Souvent, les plus âgés relataient les derniers événements marquants provoqués par le Tompuku (dessin 1), tandis que les plus jeunes préféraient évoquer les différentes couleurs de l'eau, symboles de sa vivacité. Les 

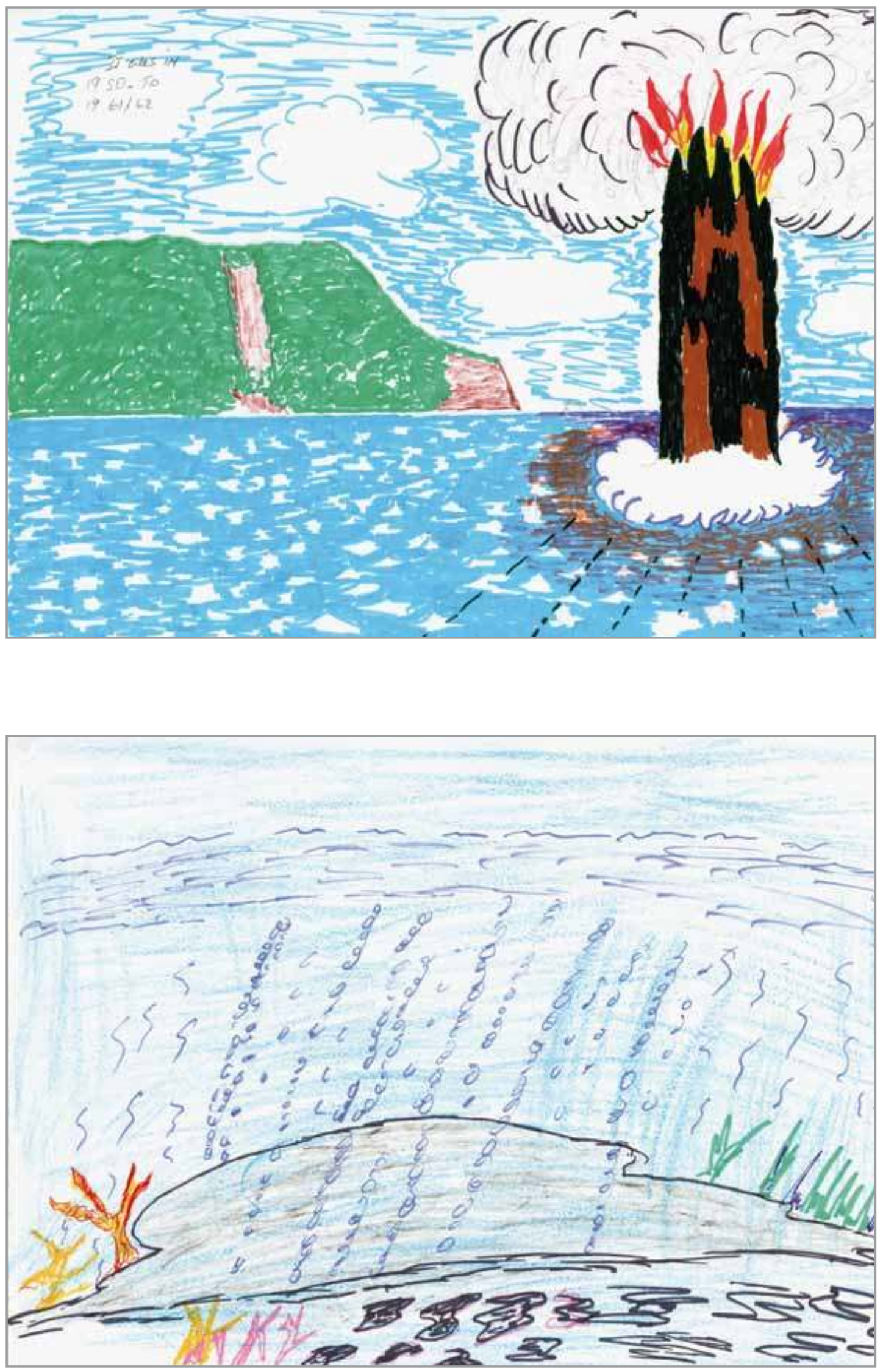
Dessin 1

« Le Tompuku en train de faire sortir

le feu et les pierres de sa bouche ",

Tapanga Toraa, homme de 54 ans,

12 février 2011, Kurumape

Le dessin est le souvenir visuel de la dernière éruption du volcan. Lorsque le magma et l'eau sont entrés en contact, il y a eu plusieurs explosions. De la côte, il était possible d'observer des pierres, de la fumée et du feu jaillir hors de l'eau.

\section{Dessin 2 \\ « Le Tompuku quand tout va bien », Hendrix Joël, homme de 22 ans, 6 mars 2011, Kurumape}

D'après le dessinateur, le volcan est « un long caillou allongé au fond de l'eau » duquel émanent des bulles, symboles de sa vivacité.

Pour les habitants les bulles signifient que le volcan respire et qu'il est encore en activité. données recueillies font état de l'utilisation de trois couleurs au-dessus de la « bouche du volcan $»^{5}$, elles ont été mentionnées puis dessinées: le bleu indiquant que le volcan « dort » et qu'il n'y a aucun danger à craindre (dessin 2); le jaune/vert, annonçant que le volcan commence à se réveiller et qu'il faut prêter une attention régulière à son activité (dessin 3); enfin, le rouge/orangé donne l'alerte, et indique qu'il faut fuir vers le sud de l'île pour s'y mettre à l'abri, car l'éruption serait imminente (dessin 4).

Ces repères visuels étaient mentionnés à travers les dessins qui permettaient dans ce contexte-ci « de contourner les difficultés du discours » comme l'observe Servane GuebenVenière à propos de la « carte mentale $»^{6}$ (Gueben-Venière, 2011 : 6). En effet, les dessins avaient une visée descriptive permettant de signifier des situations autrement qu'avec des mots et mettaient en exergue, par les couleurs choisies et les traits réalisés ce qui décrivait au mieux le volcan. Les villageois accordaient également un intérêt documentaire à cet outil, celui de rendre visibles des éléments jusque-là méconnus. Il est arrivé ainsi quelques fois, lors de discussions informelles que l'on me demande de dessiner ma maison, un jardin ou les plantes les plus cultivées en France.

\section{Une narration imagée}

Alors que l'exercice du dessin était inhabituel, voire inconnu, pour une majeure partie des personnes interrogées ${ }^{7}$, peu ont refusé de dessiner, à l'exception des femmes qui préféraient souvent s'en remettre à leur époux, prétextant ne pas savoir faire. À Tongoa et Tanna, la division sociale du travail (ou spécialisation des fonctions de chaque individu) est manifeste et maintient chacun des deux sexes dans des sphères d'action qui leur sont réservées. La lourdeur des tâches quotidiennes qui incombent aux femmes (entretien des jardins, éducation des enfants, cuisine, etc.) ne leur laisse guère de temps pour les loisirs. Le corpus recueilli est ainsi principalement composé de dessins masculins, puisque seulement cinq femmes ont accepté de dessiner. En revanche, afin de conduire des entretiens, je les accompagnais dans leurs jardins, espaces où elles passent une grande partie de leur journée et où elles pouvaient se livrer plus facilement. Pour des questions de commodité les dessinateurs ont majoritairement choisi de se prêter à l'exercice sur la table de la maison où je séjournais. D'autres, les plus âgés, ont dit préférer dessiner chez eux, afin de bénéficier de l'aide des plus jeunes auxquels ils pouvaient décrire les formes à représenter et les couleurs à employer. Je fais l'hypothèse que lorsqu'ils déléguaient ce travail, cela leur permettait d'évoquer des situations antérieures marquantes et de transmettre ainsi une mémoire, comme l'évoque Carlo Severi faisant référence à Aby Warburg à propos de son analyse des dessins d'enfants hopi, lorsqu'il explique notamment que « le rôle de l'image est constitutif du processus de transmission des connaissances » (2003: 77).

Ces séances m’ont permis d'observer en détail le processus de production des dessins en recourant à la chaîne opératoire, instrument méthodologique développé par les études de technologies culturelles (Lemonnier, Latour, 1993; Cresswell, 1996). La chaîne opératoire est définie par Robert Cresswell comme « une série d'opérations qui transforment une matière première en un produit, que celui-ci soit objet de consommation ou outil » (2010 : 26). De plus, cet outil méthodologique permet de rendre compte des différentes étapes d'un processus technique, de son état de matière première à celui d'artefact, puis 

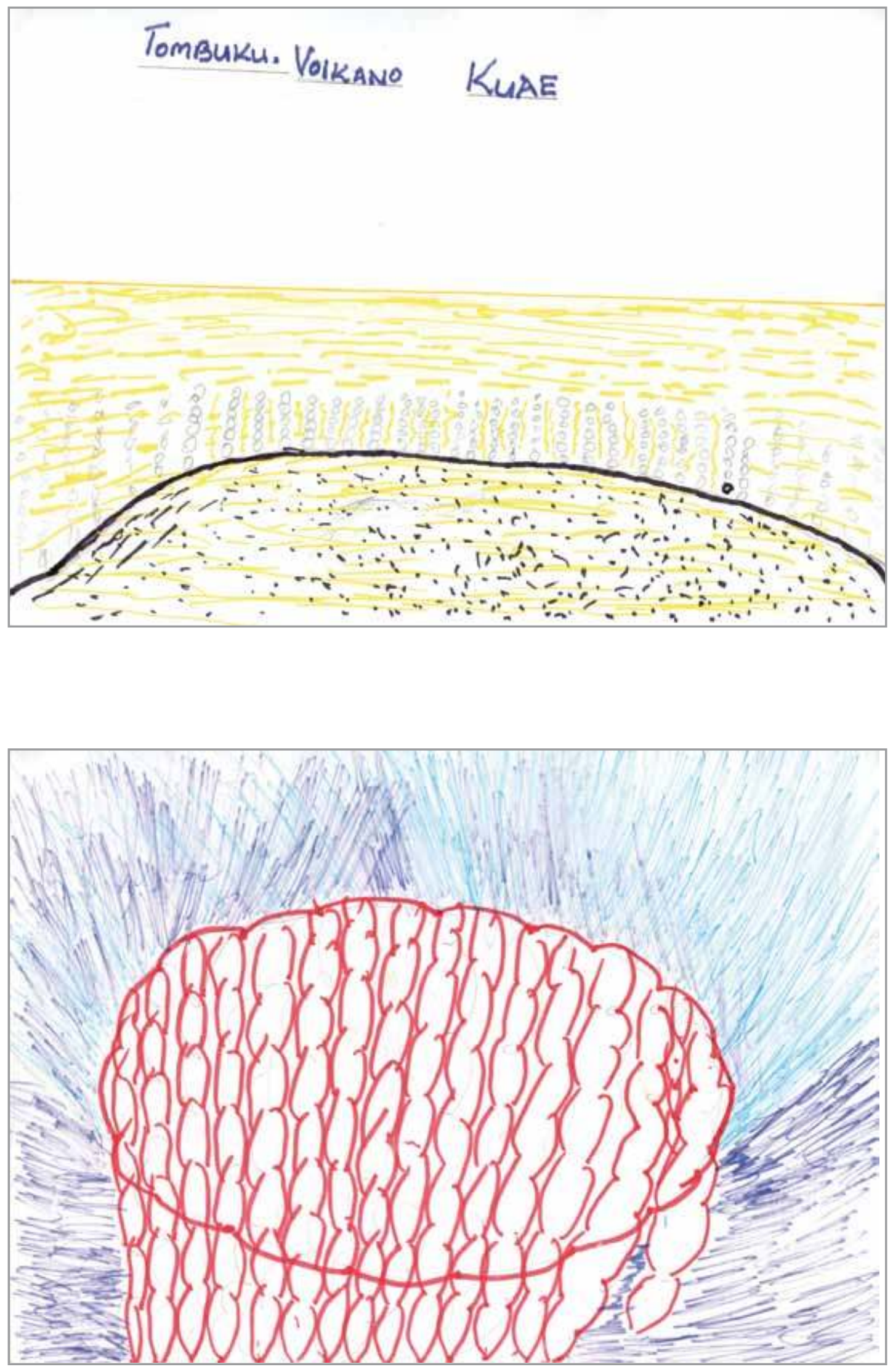

188

Maëlle Calandra 


\section{Dessin 4}

« Les bulles du Tompuku quand il est prêt à exploser », Daniel Henry, homme de 63 ans,

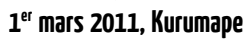

Le dessinateur a choisi de mettre en avant les bulles du volcan qui sont rougeoyantes et nombreuses avant l'éruption. Le danger d'une éruption contraint les villageois à quitter le village. de rebut. L'observation des actions de création à l'origine du dessin a permis d'établir qu'il fallait en moyenne deux heures au dessinateur pour le réaliser, et ce, en trois principales étapes. Le dessin commence par une esquisse monochrome. Cette phase est souvent la plus longue, car le dessinateur pose beaucoup de questions sur le sujet du dessin, hésite, gomme et recommence. Comme le fait remarquer César Florès, l'action graphique implique « des prises successives de décision concernant l'ordre d'exécution de ses différentes composantes [...] ce qu'il faut dessiner d'abord et ensuite, avant et après » (1985 : 186-187). Et ce, afin de pouvoir donner forme au dessin pensé et imaginé. Le choix de commencer le dessin par une esquisse résulte peut-être de l'influence scolaire. En effet, à l'école primaire, les enfants apprennent qu'il est préférable d'utiliser un intermédiaire, un brouillon, pour y formuler leurs réponses. Ce n'est qu'une fois qu'ils sont certains de leurs résultats, qu'ils les rédigent au propre dans leur cahier. Ainsi, tout comme le brouillon, l'esquisse permet de suivre le processus de production du dessin et d'accompagner la construction de la réflexion. Une fois satisfait, le dessinateur ajoute de la couleur et remplit les formes, c'est la deuxième étape. Enfin, lors de la dernière étape, il ajoute des détails de manière à préciser le lieu de la scène (sous la mer, sur l'île, au village ou dans un jardin). Le dessinateur adjoint aussi des indices sur le temps (pluie, soleil, jour, nuit) afin de pouvoir identifier la saison ou le moment de la journée.

À la différence des hommes qui employaient des couleurs proches de celles observées dans leur environnement naturel et représentant une scène qu'ils qualifiaient de « réaliste », les femmes ont souvent ajouté des motifs imaginés à leurs dessins. Ainsi, lorsqu'elles dessinaient des jardins, elles ajoutaient des fleurs multicolores (dessin 5) ou employaient des couleurs inhabituelles pour représenter un animal (dessin 6). La vache était par exemple dessinée tachetée de rouge, de blanc et de noir. Elles expliquaient vouloir rendre le dessin « joli » et accordaient une plus grande importance à la qualité esthétique de leur figuration plutôt qu'à son réalisme.

Pour saisir la réalité sociale qui s'exprime à travers ces images et analyser le choix des motifs, il est nécessaire que l'ethnologue ait une connaissance suffisante du contexte d'étude dans lequel sont produits les dessins. Aussi cela limitera-t-il les erreurs d'interprétation et de compréhension.

À Tongoa, afin d'approfondir mes observations et d'avoir une idée plus précise de la répartition des savoirs entre les générations et les genres, j’ai choisi de porter mon attention sur les enfants, en interrogeant leurs connaissances relatives au volcan, certaine qu'ils étaient informés de sa présence au fond de l'eau. Six enfants volontaires âgés de 8 à 13 ans se prêtèrent à l'exercice; je leur proposais de dessiner le Tompuku. Dans un premier temps, tous restèrent interloqués et étonnés par la consigne. Pensant que cela venait de mon bislama ${ }^{8}$ et de mon mauvais accent, je reformulais mes indications. Finalement, le plus âgé acquiesça et commença à esquisser un paysage; les autres l'observèrent et l'interrogèrent en nakanamanga ${ }^{9}$ (langue locale), puis se mirent à dessiner un volcan à forme conique dans l'eau, le même, le Lopevi ${ }^{10}$ (dessin 7 ). Tous représentèrent ce volcan très actif dont le panache de cendres est observable du village de Kurumape (Tongoa). Les enfants ignoraient la présence du Tompuku, pourtant situé aux abords de l'île. Puisqu'ils ne connaissaient pas le volcan auquel je faisais référence, ils dessinèrent celui qui leur était le plus familier. À l'école primaire, l'exemple du Lopevi est étudié lors des leçons introductives sur l'activité volcanique du pays ${ }^{11}$. Cette observation permit d'engager des entretiens sur la transmission des savoirs entre les générations et la répartition des savoirs en fonction du genre. Les hommes expliquèrent alors que le « cerveau » des enfants interrogés n'était « pas assez développé ». 

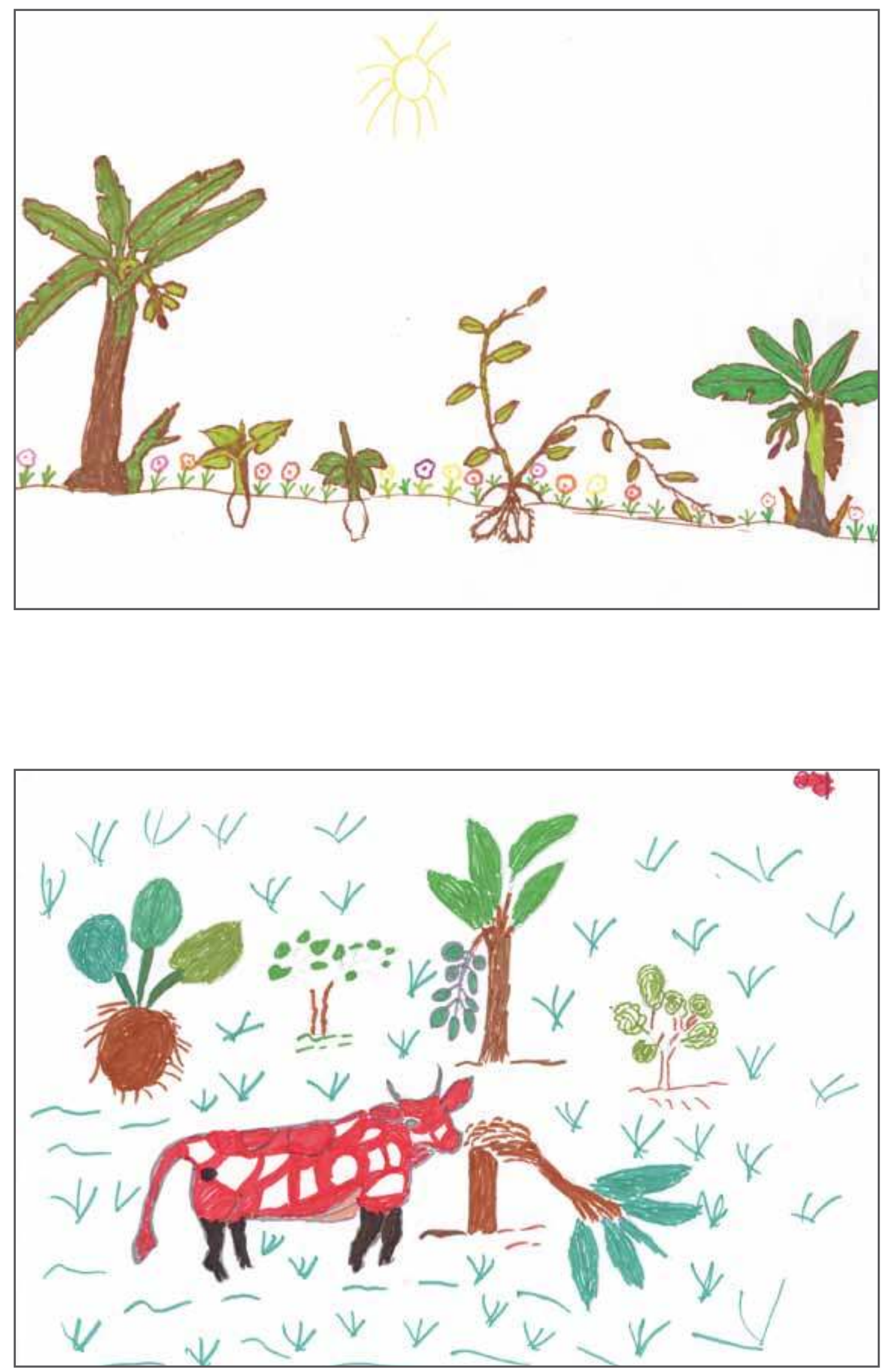
Dessin 5

"Le jardin quand tout va bien » Marie-Rose Yarris, femme de 28 ans,

Lamlu

Cette femme a dessiné son jardin en insistant sur la forme des feuilles afin que la plante soit facilement identifiable (bananier, taro, igname). En revanche pour rendre le dessin « joli », elle a ajouté de nombreuses fleurs multicolores et inexistantes dans son jardin.
Ce sont les hommes qui transmettent aux plus jeunes dont le « cerveau » est considéré comme « fini ». C'est-à-dire quand l'enfant est suffisamment mature pour comprendre l'histoire coutumière justifiant la présence du volcan au fond de l'eau, et qu'il est capable de la retenir pour la transmettre à son tour, le moment venu. Outre l'âge de l'enfant, la transmission ne se fait que si le contexte est favorable, à savoir lorsqu'un événement soudain, tel un séisme est venu rappeler la proximité du volcan avec les habitants. Ainsi, lors du dernier grand tremblement de terre survenu à Tongoa le 29 mai 2009, pour rassurer les enfants effrayés par les secousses, les hommes ont désigné le responsable en racontant l'histoire coutumière du Tompuku.
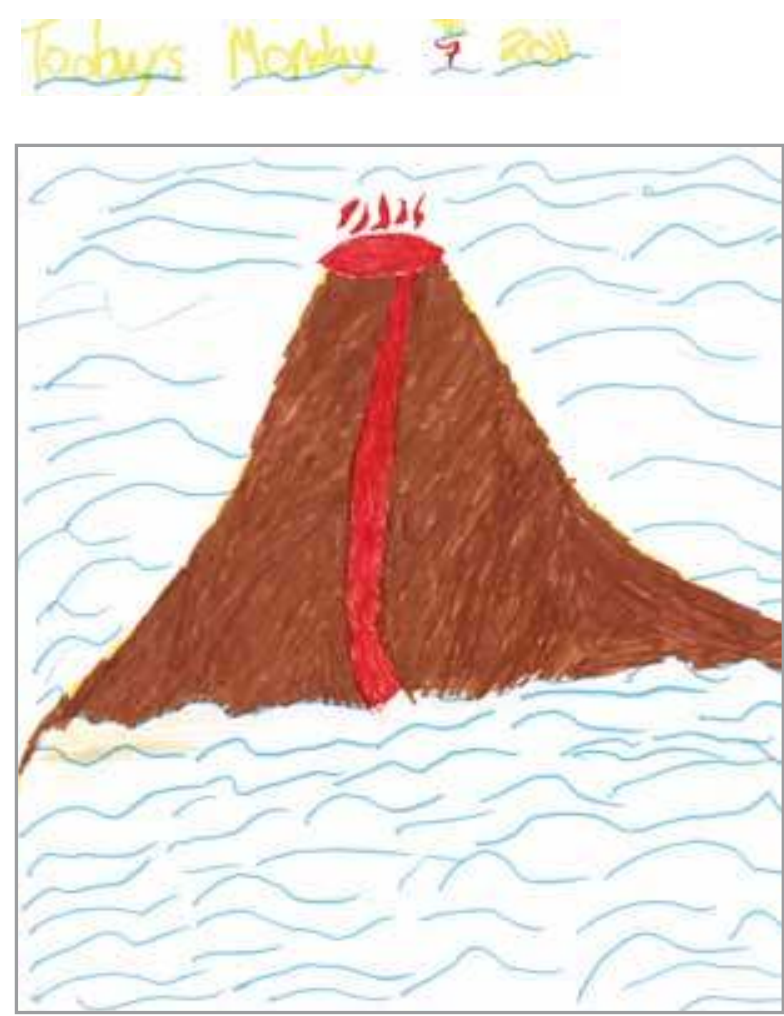

Dessin 6

"Le jardin détruit par une vache », Rosema Nako, femme de 30 ans, 18 mars 2012, Lamlu

La dessinatrice a choisi d'insister plus sur la vache que sur les végétaux. Les bananiers et les taros sont coloriés avec des couleurs observées dans les jardins, tandis que les motifs de l'animal et ses couleurs ont été inventés pour rendre le dessin « plus beau » et mettre ainsi l'animal au premier plan. 

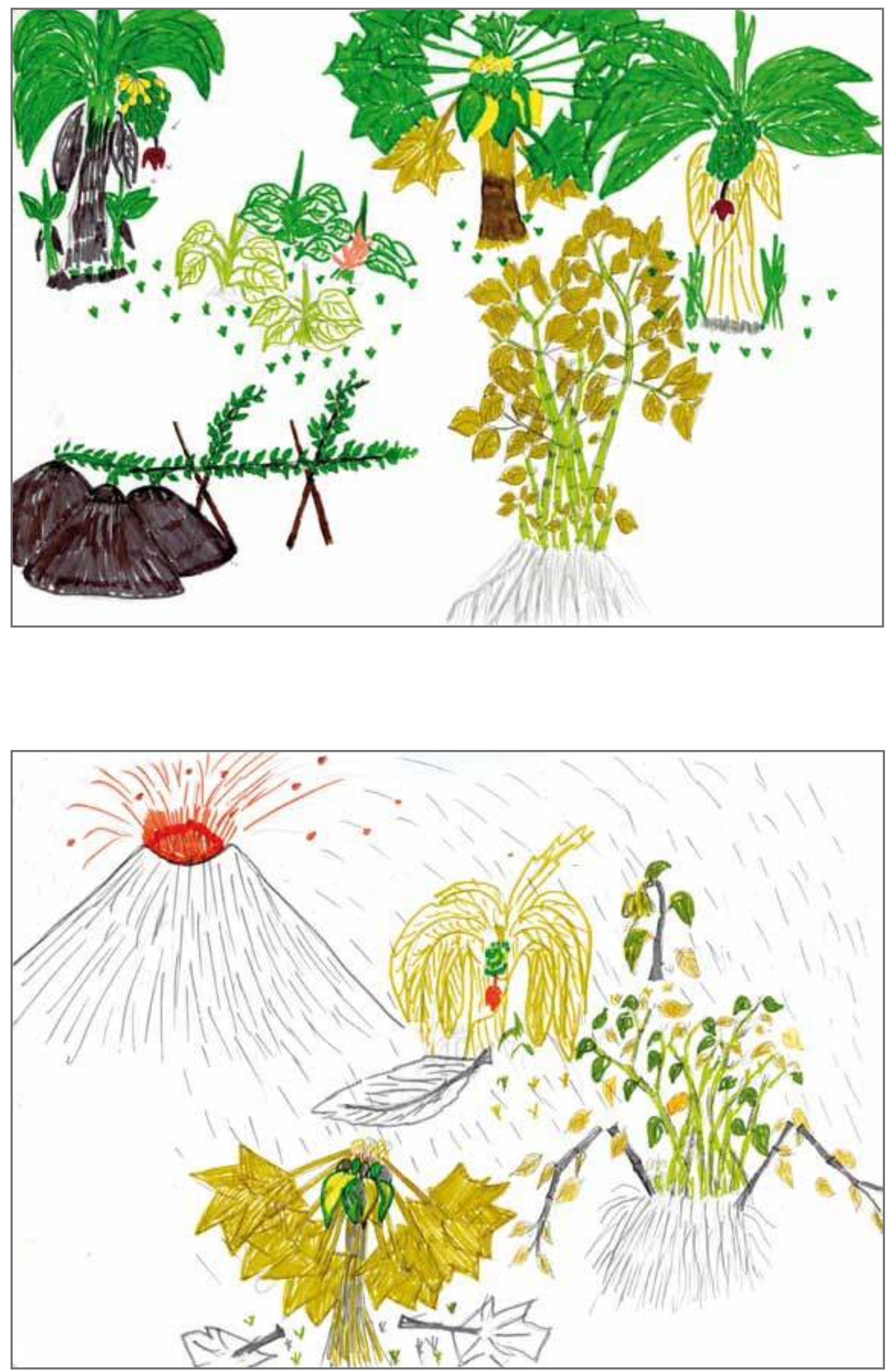
"L Le jardin de cultures mélangées lorsque tout va bien », Xavier Tao, homme de 19 ans, 6 janvier 2012, Lamlu

Au premier plan sont dessinés des buttes d'ignames et un kava, au second plan il y a des bananiers, un papayer et au centre des taros. L'organisation du dessin renvoie à celle des jardins où les arbres fruitiers sont sur les côtés afin de ne pas faire d'ombre aux cultivars et de délimiter l'essart. Par les couleurs employées et la précision du dessin, le dessinateur a voulu mettre en avant la bonne santé du jardin.

\section{Dessin 9}

« Le même jardin après plusieurs jours de pluie de cendres du Yasur ", Xavier Tao, homme de 19 ans, 7 janvier 2012, Lamlu

Ici, l'élément principal du dessin est le volcan Yasur. Lorsque le vent souffle ses cendres acides dans les jardins, les feuilles des végétaux sont brûlées et asphyxiées, mettant à mal les récoltes. Pour le dessinateur ceci constitue l'un des événements le plus dommageable qui puisse survenir dans ses essarts.

\section{Un médium graphique}

Recourir au dessin permet de mieux cerner son objet d'étude et parfois de mieux identifier les personnes auprès desquelles il est important de conduire les entretiens. Appréciant la portée heuristique de cette technique d'enquête, je la mis de nouveau à profit lors d'un second travail ${ }^{12}$ ethnographique en 2012, à Lamlu, un village du Centre Brousse de l'île de Tanna. L'objectif de ce projet fut de s'intéresser aux rapports techniques et symboliques qu'entretiennent les habitants avec leur environnement naturel et plus particulièrement aux jardins de subsistance, espaces où la nature est façonnée selon des processus horticoles particuliers. À Tanna, comme ailleurs au Vanuatu, les jardins de subsistance répondent à des besoins alimentaires, rituels, et d'échanges entre lignages. Leur destruction peut entraîner des perturbations importantes dans la vie quotidienne des habitants. Le disasta ${ }^{13}$ (désastre en bislama) se mesure à l'étendue des dommages dans ces espaces. Il fut proposé à l'issue d'un premier entretien sur les jardins que les horticulteurs dessinent leurs essarts avant et après un événement destructeur (celui de leur choix). La représentation mentale matérialisée par le dessin a rendu possible l'appréhension de la façon dont l'aléa (cyclone, séisme, cendre volcanique...) peut détériorer les jardins. L'interprétation faite sur le dessin par les interlocuteurs de l'étendue des dégâts, a permis de cataloguer les désastres et l'illustration qui a été réalisée des événements les plus dommageables, a facilité les échanges. À partir du corpus collecté, j’ai retenu trois critères pour analyser les détails des dessins: le choix des plantes représentées, les couleurs employées et le dessin lui-même (les traits, les espaces laissés vides, la correspondance avec les situations évoquées durant les entretiens). Plusieurs fois, le choix du disasta a été motivé par la volonté de décrire une situation à laquelle l'ethnographe n'avait pas assisté. Les dessinateurs ont proposé que leurs dessins soient considérés comme des photos, comme des témoignages, constituant le souvenir d'un événement passé.

De manière à rendre explicites les dégâts engendrés par la catastrophe, j'ai suggéré que deux dessins soient réalisés. L'un évoquant le jardin lorsque « tout va bien » (dessins 8, 10, 12, 14) et l'autre après l'événement destructeur (dessins 9, 11, 13, 15). L'observation des détails représentés sur les dessins avant que l'incident ne survienne révèle l'attachement des interlocuteurs à souligner la grande variété des espèces cultivées dans les jardins, tout comme ils le faisaient lors des entretiens. Sur la plupart des illustrations, il est possible de distinguer plusieurs variétés de végétaux grâce à la couleur des tiges et des feuilles. Les plus représentés sont les taros, les kavas, et les bananiers qui sont également les espèces les plus consommées et les plus utilisées lors d'événements coutumiers. Elles sont majoritairement dessinées en vert et constituent un ensemble « réaliste », puisqu'identifiable pour quiconque ne serait pas informé du thème du dessin.

À ces dessins s'opposent les illustrations où figure la catastrophe. Les traits y sont moins précis, les plantes moins reconnaissables et des zones sont laissées vierges. Ces caractéristiques symbolisent les bouleversements engendrés par le passage d'un cyclone ou le dépôt des cendres du Yasur dans les parcelles cultivées. Ces figurations correspondent aux descriptions entendues lors des entretiens antérieurs. Les végétaux décrits comme étant les plus sensibles aux rafales des cyclones tels que les bananiers et les maniocs sont systématiquement dessinés par les illustrateurs. L'événement qui est à l'origine de ces conséquences est rendu intelligible par des détails l'évoquant. Ainsi, lorsque la catastrophe 

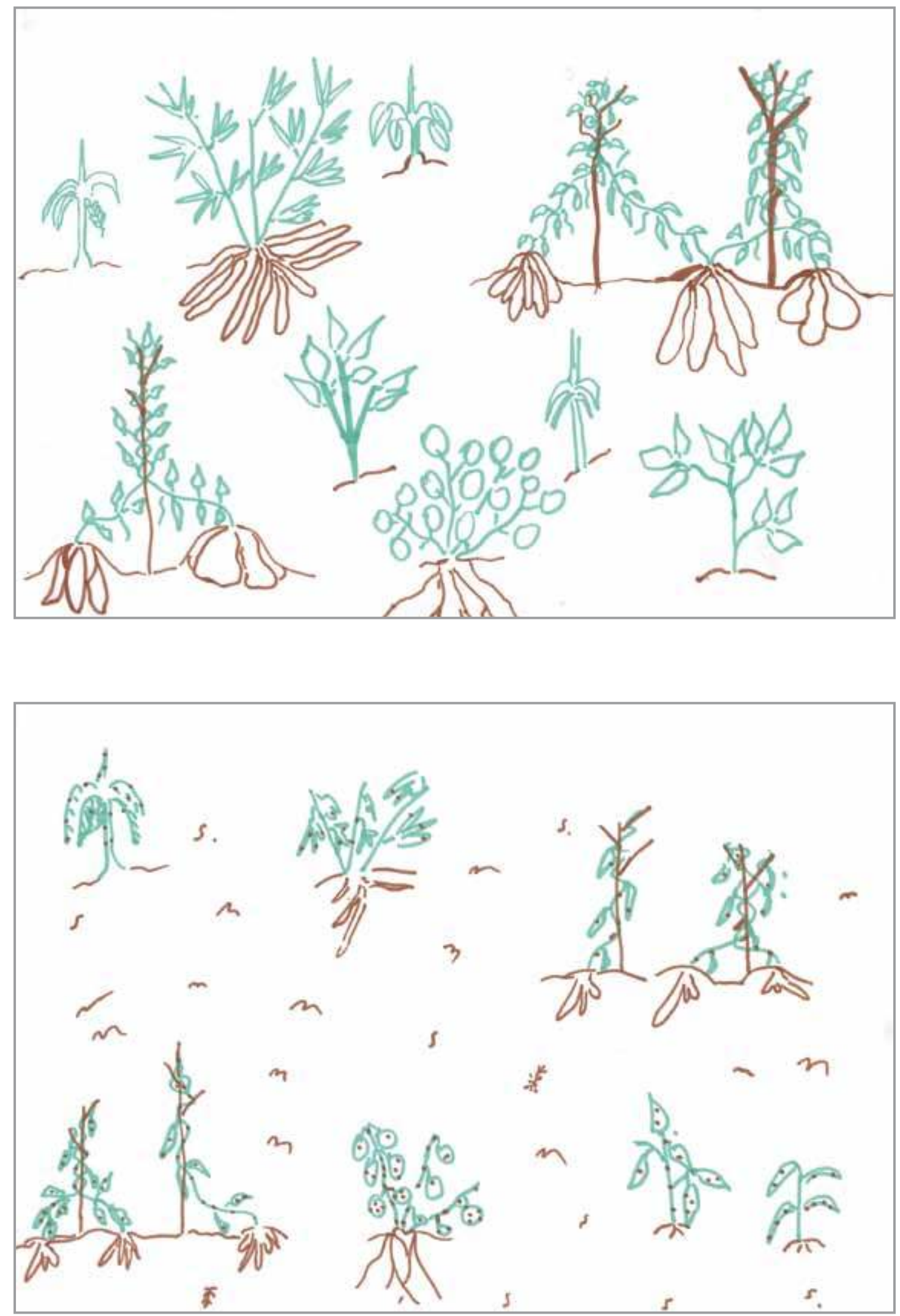
est un cyclone, ce dernier est représenté par des traits horizontaux allant tous dans une Pour signifier la bonne santé du jardin, les tubercules d'ignames et de taros sont dessinés longs et nombreux, ils semblent être arrivés à terme.

\section{Dessin 11 \\ « Le jardin après le passage d'une femme ayant ses menstrues $"$, Jacques Lamoul, homme de 35 ans, 4 janvier 2012, Lamlu}

Pour illustrer cet aléa qu'il redoute, le dessinateur a représenté des feuilles malades et abîmées. Les tubercules ont disparu, elles ont pourri et ne pourront pas être consommées. même direction et les plantes sont toutes couchées à terre de la même manière. Quand il s'agit de signifier les effets des cendres volcaniques, le volcan et une pluie noire sont dessinés. Il est notable que les plantes les plus résistantes à l'aléa représentées sur le premier dessin disparaissent dans le second. La place est en effet laissée à ce qui a marqué la mémoire de l'horticulteur et ce qui, pour lui, signifie la catastrophe. Par conséquent, le taro et la patate douce puisqu'ils se développent près du sol et qu'ils ont une faible prise au vent, n’apparaissent pas. Ils sont moins représentatifs des conséquences d'un disasta. Les maniocs et les bananiers sont en revanche plus sensibles et sont ainsi souvent dessinés. Ils constituent le souvenir mnésique du passage d’un événement destructeur. Les détails du dessin illustrent l'événement et mettent en exergue ce qui permet d'identifier le sinistre représenté par les traits, les motifs et les couleurs. Par exemple, le noir est la couleur dominante d'un dessin représentant les cendres acides du volcan qui annihilent les récoltes. Et ce, car cette couleur est associée au volcan et à ses cendres. De plus, le volcan qui n'est pourtant pas visible des jardins est dessiné pour marquer son implication dans le désastre. Il en va de même pour les illustrations signifiant les incursions dévastatrices des cochons ou des vaches dans les jardins. En effet, alors que l'animal est rarement pris en flagrant délit, il est presque toujours représenté sur l'image, pour qu'au premier regard, l'événement destructeur soit identifiable.

En dépit des nombreuses entrevues sur les jardins et les événements pouvant mettre en péril ces espaces, seul le dessin a permis de libérer la parole des hommes. Par ce média, ils ont en effet évoqué une calamité redoutée et qu'ils considèrent comme étant la plus préjudiciable: le sang menstruel (dessins 10 et 11). Selon les hommes ce disasta a des effets invisibles dans un premier temps, plusieurs jours ou semaines sont nécessaires avant de pouvoir remarquer qu'une femme ayant eu ses menstrues est venue dans la parcelle cultivée. Pour cela, il faut que les tubercules soient déterrés, ils sont alors constatés pourris et jaunis, signe d'un développement végétatif anormal. Ceci peut également être indiqué par l'odeur putride s'échappant subitement du sol. De manière à épargner les plantes cultivées des dangers que représente ce sang, les femmes doivent respecter un ensemble de prescriptions et d'interdits. Comme l'ont expliqué les horticulteurs, lorsqu'une femme est indisposée, il lui est formellement interdit de se rendre dans les nouveaux jardins où poussent des taros et des kavas, car leurs rhizomes sont très sensibles à ce sang, et son odeur putride entraîne leur dégradation. La femme ne doit avoir aucun contact avec la nourriture, car elle pourrait la contaminer et rendre les hommes malades (céphalées, fièvre, fatigue). Ainsi, durant cette période les femmes ont dit ne pas cuisiner et ne touchent à aucun ustensile culinaire. Elles ne se préoccupent que de leurs affaires personnelles pour «protéger les autres». Pourtant, comme l'ont expliqué les hommes, ce flux est difficilement contrôlable, car son odeur est colportée dans les jardins par le vent, ce qui engendre la souillure des plantes. Les dessins ont également été l'occasion de m'informer qu'il serait mal venu que j'approche mes interlocuteurs ou que je me rende dans les jardins à l'apparition de cette « substance dangereuse » (Coupaye, 2009: 61).

Au cours de l'enquête, aborder ce phénomène destructeur à travers l'illustration graphique a facilité les échanges et a limité la gêne des hommes lorsqu'ils évoquaient ce sujet. Je fais ici la supposition qu'à Tongoa et Tanna la représentation graphique, introduite nouvellement de l'extérieur, n'est soumise à aucun interdit explicite. Ce qui ne peut être formulé oralement, peut l'être graphiquement. C'est en cela que le dessin constitue 

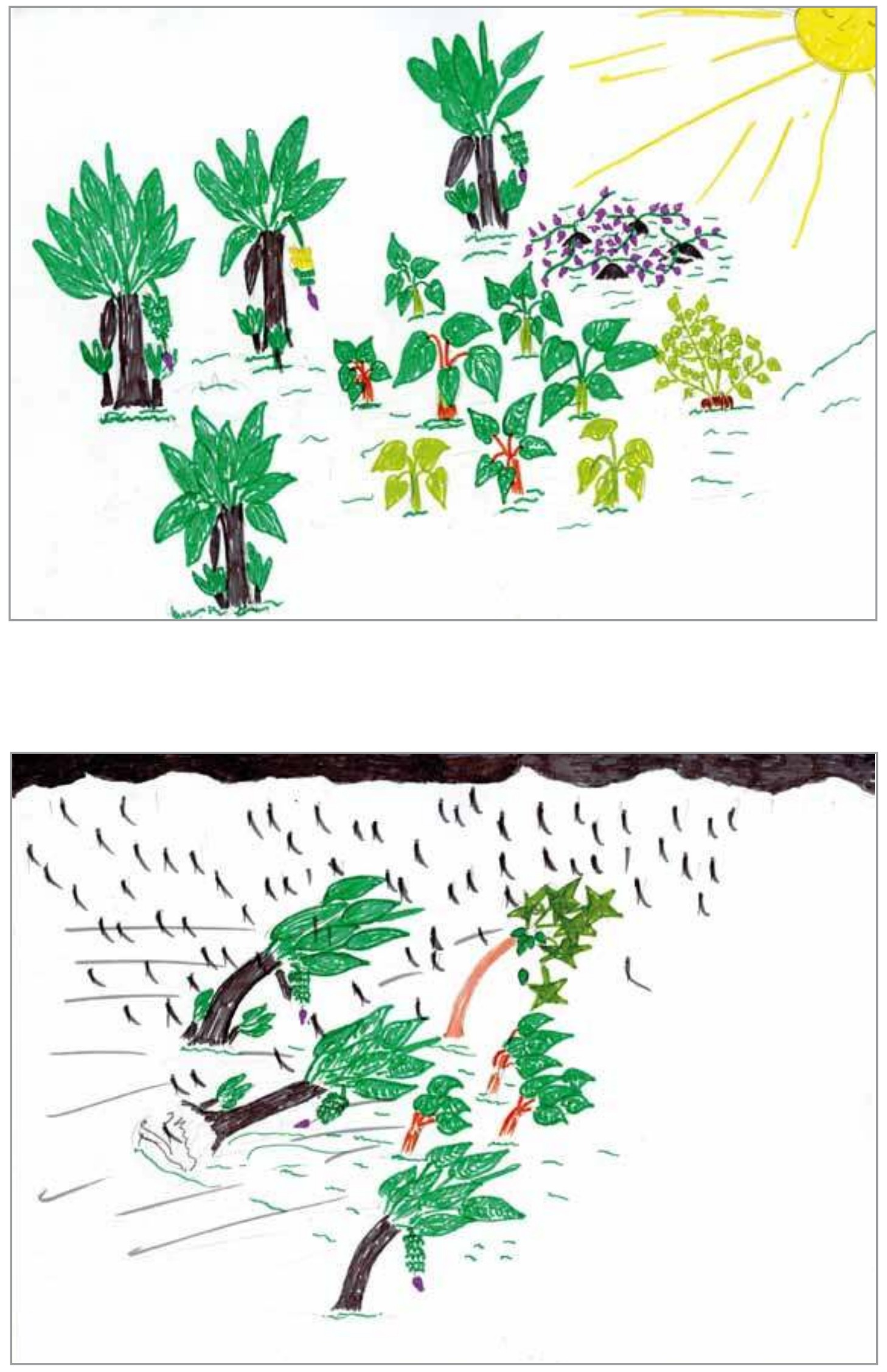
Dessin 12

"Le jardin au mois de mars », Jean-Baptiste Yaris, homme de 23 ans, 16 mars 2012, Lamlu

Au centre du dessin sont représentées différentes variétés de taros, un kava et des ignames, soit les espèces les plus importantes pour cet homme. Aux abords du jardin se trouvent différents bananiers; sous un soleil bienveillant, les plantes prospèrent. un objet interface, libérateur de paroles. Ceci rejoint les conclusions du travail de la psychanalyste Sophie Morgenstern (1937) qui considère la valeur expressive du dessin supérieure à celle du langage. À ce propos, Francastel déclarait:

« Les hommes ont exprimé à travers les âges leur perception, c'est-à-dire leur sensibilité et les valeurs qui s'attachent aux représentations qu'ils se font du monde extérieur, à travers une quantité d'activités qui échappent au contrôle - aux lois - du langage [...] » $(1970$ : 35).

Dans un contexte où le dessin est une pratique nouvelle, introduite par l'ethnographe, il semble maintenant essentiel de mesurer les modalités de production du dessin (le poids des astreintes matérielles et techniques) mais également les motivations du dessinateur ou l'influence de la consigne du chercheur sur la figuration.

\section{$\&$}

Au terme de cet article il est possible d'avancer que le dessin, appliqué à des objets de nature, a plus qu'une fonction illustrative. Il constitue un instrument de médiation capable de libérer la parole lorsqu'un sujet dont on ne parle pas ou peu localement est abordé, tel le sang menstruel dont les conséquences dans les essarts sont analogues à celles d'événements physiques. Cette technique de transcription graphique permet la mise en évidence de détails nouveaux et essentiels à la progression de la réflexion. Elle facilite l'objectivation et la réorientation de l'enquête au gré des dessins collectés. Comme l'a écrit Albert Piette à propos de l'image photographique, l'image figurative constitue un moyen d'objectivation de l'écriture original, permettant d'accroître « la dimension réflexive de la recherche » (2007 : 26). En outre, le dessin constitue un nouvel outil pour analyser les rapports entre la pensée verbale et la « pensée figurative » (Francastel, 1965) par la structure cosmologique qu'il laisse transfigurer. Il serait peut-être possible d'établir « une raison graphique » reflet d'une «pensée visuelle » à partir des dessins dont on a tenté ici de démontrer l'intérêt.

\section{Dessin 13}

« Le jardin pendant un cyclone », Jean-Baptiste Yaris, homme de 23 ans, 16 mars 2012, Lamlu

Le vent violent contraint les plantes à se courber, déterre les bananiers et les fruits tombent. Le ciel est noir et les gouttes de pluies épaisses. Tout indique que la situation est critique pour le jardin. Les plantes détruites vont pourrir et ne pourront être consommées. 
Dessin 14

« Le jardin pendant la saison des pluies ", Charley Nako, homme de 63 ans, 10 janvier 2012 ,

Lamlu

Au premier plan du dessin se trouvent des buttes d'ignames parmi d'autres végétaux, tels que des patates douces et des taros. Des arbres fruitiers bordent le jardin. Par le nombre d'espèces cultivées, le dessinateur a voulu signaler la richesse de son jardin.

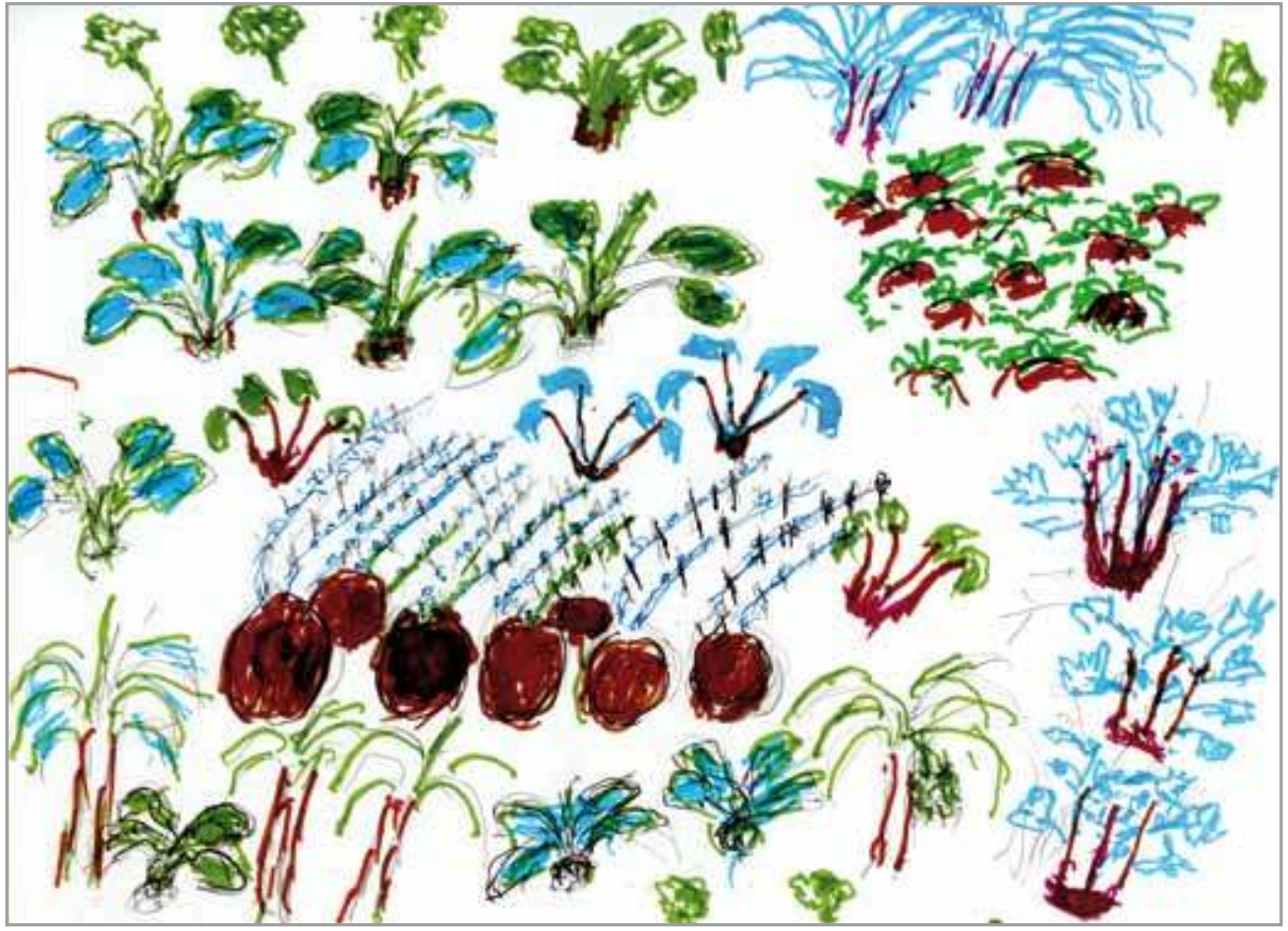




\section{NOTES}

1. Les termes de nature et d'anthropologie de la nature sont employés en référence au travail de Philippe Descola, qui traite des façons qu'ont les cultures de penser et de socialiser les rapports entre humains et non-humains. Nos usages et notre rapport à l'environnement naturel influencent nos perceptions et notre compréhension de celui-ci. Le dualisme propre à l'Occident n'est pas universel, mais relatif et ne serait qu'une cosmologie parmi d'autres (Descola, 2005 : 25).

2. Les illustrateurs des dessins collectés sont âgés de 5 à 82 ans. Au cours de terrains à Tongoa en 2011, 61 dessins ont été collectés et 39 lors de mon séjour à Tanna en 2012, ce qui constitue un total de 100 . Parmi ces dessins, 28 ont été réalisés par des enfants et 5 par des femmes.

3. La ceinture de feu ou « ring of fire » concentre plus de $70 \%$ de l'activité volcanique mondiale: <http://www. mpl.ird.fr/suds-en-ligne/fr/volcan/vanuatu/vanuat01. htm> consulté le 3 septembre 2013.

4. Chaque année, le Vanuatu compte en moyenne deux cyclones et plusieurs dépressions tropicales. Informations issues du site internet: <http://www. meteo.gov.vu/TropicalCyclones/PrecautionaryAdvice/ tabid/175/Default.aspx> consulté le 3 septembre 2013.

5. Les habitants attribuent des caractéristiques humaines au volcan: la caldera est considérée comme étant sa bouche et parfois son œil et les zones sur l'île où la terre est chaude, son nez.

6. Ce concept a été introduit par Edward Tolman (1948) suite à une expérience réalisée avec des rats qui devaient s'orienter dans un labyrinthe. La carte mentale ou cognitive map traduit de manière visuelle les représentations mentales de l'être humain ou d'animaux quant à l'organisation de l'espace dans lequel ils évoluent.
7. Dans ces deux îles, les enfants étaient assez familiarisés avec cette activité puisqu'à l'école il arrive que les enseignants proposent des séances de dessins pour illustrer une chanson, une poésie ou pour décorer les murs de la classe.

8. Le bislama est apparenté aux pidgins mélanésiens (le tok pisin de Papouasie-Nouvelle-Guinée et le pijin des îles Salomon). D'abord « code inter-ethnique d'usage vertical entre colonisateurs européens et natifs des îles », son usage a complètement changé après l'indépendance en devenant « la langue la plus importante de la République » (Charpentier, 2009 : 219).

9. Le nakanamanga est parlé au nord-ouest de l'île de Tongoa, au nord d'Efate (île principale du Vanuatu, où se trouve la capitale du pays Port-Vila) et dans des îles limitrophes. Il est aussi appelé ngounéen ou nord-efatois (Siméoni, 2009 : 76).

10. Lopévi est une île-volcan, située au nord de Tongoa. Elle est inhabitée depuis les années 1960 en raison des fréquences éruptives du volcan trop dangereuses pour la population. À présent, seuls les pêcheurs s'y rendent.

11. Dans l'ouvrage Histri Blong Yumi Long Vanuatu, matériel pédagogique pour les enseignants de l'archipel, le Lopévi est pris pour exemple dans un chapitre dédié à la formation volcanique du pays (Lightner $\&$ Naupa, 2005 : 34).

12. Travail de terrain réalisé dans le cadre de l'ANR-2010STRA-011-05, intitulée « Mesure des performances de systèmes complexes à base de plantes à multiplication végétative en zone tropicale humide [Végé-Culture] ».

13. Mot formé à partir de l'étymon anglais disaster introduit récemment dans le lexique du bislama.

\section{POUR CITER CET ARTICLE}

Calandra, M. 2013 Faire dessiner le terrain. La nature à « risques » et les jardins de subsistance à Tanna et Tongoa (Vanuatu), in Guy, H. Jeanjean, A. \& A. Richier Le Cadavre en procès, TechniquesE Culture 60 : $182-201$. 


\section{RÉFÉRENCES}

Aumont, J. 2005 [1990], L'image, 2e édition. Paris: Nathan.

Becker, H. S. 2001 «Sociologie visuelle, photographie documentaire et photojournalisme », Communications 71 : 333-351.

Coquet, M. 2009 Lalbum de dessins indigènes. Thérèse Rivière chez les Ath Abderrahman Kebèche de l'Aurès (Algérie), Gradhiva 9: 188-203.

Charpentier, J.-M. 2009 Les Langues. In P. Siméoni Atlas du Vanouatou (Vanuatu). Port-Vila: Éditions Géo-Consulte.

Coupaye, L. 2009 Décrire des objets hybrides, Techniques \& Culture « Technologies » 52-53: 61, mis en ligne le 19 juillet 2012, consulté le 6 septembre 2013. URL: http://tc.revues.org/4730

Cros, M. 1993 La Maladie dessinée ou la maîtrise de l'infortune. In M. Fiéloux, J. Lombard, J.-M. Kambou-Ferrand (Dir.), Images d'Afrique et sciences sociales: les pays lobi, birifor et dagara: actes du colloque de Ouagadougou (10 au 10 décembre 1990). Paris: ORSTOM; Karthala: 295-310.

Cresswell, R. 2010 Techniques et culture: les bases d'un programme de travail, Techniques \& Culture «Cultures matérielles » 54-55 (1): 21-45.

- 1996, Prométhée ou Pandore? Propos de technologie culturelle. Paris: Kimé.

Descola, P. 2006 La Fabrique des images, Anthropologie et Sociétés 30 (3) : 167-182.

— 2005 Par-delà nature et culture. Paris : Gallimard (Bibliothèque des sciences humaines).

Florès, C. 1985 Pensée figurative, représentation mentale et langage: l'influence de l'ordre d'énonciation des objests et de la réversibilité empirique des actions sur l'organisation temporelle du dessin, L'Année psychologique 85 (2): 185-202.

Francastel, P. 1970 Études de sociologie de l'art. Paris: Denoël et Gonthier.

— 1967 La Figure et le lieu. L'ordre visuel du Quattrocento. Paris: Denoël et Gonthier.

— 1965 La Réalité figurative. Paris: Denoël et Gonthier.

Gueben-Venière, S. 2011 En quoi les cartes mentales, appliquées à l'environnement littoral, aident-elles au recueil et à l'analyse des représentations spatiales? EchoGéo 17. URL: http://echogeo.revues.org/12573 consulté le 10 septembre 2013.

Haddon, A. C. 2010 [1935] Reports of the Cambridge Anthropological Expedition to Torres Strait. Cambridge: Cambridge University Press.

Kilani, M. 2007 La Certitude de la catastrophe. Un éclairage anthropologique. In Ch. Delécraz \& L. Durussel (Dir.) Scénario catastrophe. Genève: MEG: 45-55.

La Rocca, F. 2007 Introduction à la sociologie visuelle, Sociétés 95 : 33-40.

Lemonnier, P., Latour, B. (Dir.) 1993 L'Intelligence des techniques. Paris: La Découverte.

Lightner, S., Naupa A. (eds.) 2005 Histri Blon Yumi Long Vanuatu: Vanuatu-History-Study and teaching, Vol 1. Port-Vila: Vanuatu Cultural Centre.

Malinowski, B. 2010 [1922] Les Argonautes du Pacifique Occidental, tr. fr. Paris: Gallimard, (1 éd. en langue anglaise).

Morgenstern, S. 2003 [1937] Psychanalyse infantile. Symbolisme et valeur clinique des créations imaginatives chez l'enfant - La psychanalyse et son rôle dans l'hygiène mentale, Euvres, t. 2. Paris: Tchou, Bibliothèque des introuvables.

Pagezy, H., Carrière, S., Sabinot, C. (Dir.) 2010 Nature du monde. Dessins d'enfants. Paris: CTHS.

Piette, A. 2007 Fondements épistémologiques de la photographie, Ethnologie française 37: 23-28.

Severi, C. 2003 Warburg anthropologue ou le déchiffrement d'une utopie. De la biologie des images à l'anthropologie de la mémoire, L'Homme 165 (2003/1): 77-128.

Siméoni, P. (dir.) 2009 Atlas du Vanouatou (Vanuatu). Port-Vila: Éditions Géo-Consulte.

Soukup, M. 2011 A visualization and representation of the culture in Yawan, Papua New Guinea: Drawings in the context of a visual anthropology, Anthropologia Integra $2: 42-51$.

Tolman, E. C. 1948 Cognitive maps in rats and men, Psychological Review 55: 189-208.

Vidal, L. 2010 Faire de l'anthropologie: santé, science et développement. Paris: La Découverte. 


\section{RÉSUMÉ}

Faire dessiner le terrain. La nature à " risques " et les jardins de subsistance à Tanna et Tongoa (Vanuatu). Cet article examine les apports du dessin dans le cadre d'une enquête en anthropologie de la nature. Pour ce faire, il s'appuie sur une centaine de dessins recueillis en 2011 et 2012 à Tongoa et Tanna, îles volcaniques du Vanuatu. Les insulaires ont accepté de représenter les différents événements destructeurs auxquels leur environnement naturel est régulièrement exposé (cyclone, tremblement de terre, éruption volcanique, animaux et ménorrhées). Le dessin laisse apparaître les représentations que se font les informateurs du monde qui les entoure et permet de contourner les difficultés du discours lorsque les sujets abordés occasionnent la gêne. Il permet également l'émergence d'interactions nouvelles et essentielles à l'avancement du projet ethnographique.

\section{ABSTRACT}

To Make drawing the field. Risky nature and subsistence gardens of Tongoa and Tanna (Vanuatu). This paper would like to reflect on the contributions of picture drawing within the framework of ethnographic research about various aspects of nature. It uses a corpus of hundred of collected drawings during the years 2011 and 2012, on Tongoa and Tanna, two volcanic islands of Vanuatu. The villagers have accepted to draw their natural environment which can be disrupted or wiped out by frequent cyclical and life-threatening events (cyclones, earthquakes, volcanic eruptions, animals, menstruations). This figurative technique lets the peoples' representations about their environment appear and makes the discussion easier when speaker is embarrassed by the evoked subject. Moreover, it allows new essential interactions with the fieldwork, which improves the progress of the ethnographic research.

\section{MOTS CLÉS}

dessin, ethnographie, jardin de subsistance, volcan sous-marin, catastrophe, Vanuatu

\section{KEYWORDS}

drawing, ethnographic tools, subsistence gardens, disaster, Vanuatu 